\title{
Clifford index for reduced curves
}

\author{
Marco Franciosi ${ }^{1}$
}

Received: 17 September 2018 / Accepted: 13 April 2019 / Published online: 27 April 2019

(c) Fondazione Annali di Matematica Pura ed Applicata and Springer-Verlag GmbH Germany, part of Springer Nature 2019

\begin{abstract}
We extend the notion of Clifford index to reduced curves with planar singularities by considering rank 1 torsion-free sheaves. We investigate the behaviour of the Clifford index with respect to the combinatorial properties of the curve, and we show that Green's conjecture holds for certain classes of curves given by the union of two irreducible components.
\end{abstract}

Keywords Algebraic curve · Clifford index · Green's conjecture

Mathematics Subject Classification $14 \mathrm{H} 20 \cdot 14 \mathrm{C} 20 \cdot 14 \mathrm{H} 51$

\section{Introduction}

The Clifford index for smooth curves was introduced by Martens in [15] (see also [12]), and many authors have investigated its relation with the geometry of smooth curves. If $C$ is a smooth curve and $\mathcal{L}$ is an invertible sheaf, then the Clifford index of $\mathcal{L}$ is $\operatorname{Cliff}(\mathcal{L})=$ $\operatorname{deg}(\mathcal{L})-2 h^{0}(C, \mathcal{L})+2$ and the Clifford index of $C$ is

$$
\operatorname{Cliff}(C)=\min _{\mathcal{L} \in \operatorname{Pic}(C)}\left\{\operatorname{deg}(\mathcal{L})-2 h^{0}(C, \mathcal{L})+2: h^{0}(C, \mathcal{L}) \geq 2, h^{1}(C, \mathcal{L}) \geq 2\right\}
$$

For a smooth curve, one always has $\operatorname{Cliff}(C) \geq 0$, with equality holding only for hyperelliptic curves. Similarly, $\operatorname{Cliff}(C)=1$ if and only if $C$ is trigonal or plane quintic, and $\operatorname{Cliff}(C)=2$ if and only if $C$ is tetragonal, or plane sextic (see [16] for a thorough analysis). Indeed, the Clifford index is closely, but not completely, related to the gonality since by [7] one has $\operatorname{Cliff}(C)+2 \leq \operatorname{gon}(C) \leq \operatorname{Cliff}(C)+3$.

Caporaso in [4] studied the Clifford index of invertible sheaves on semistable curves finding interesting connections with the combinatorial properties of the curve and pointing out the problems that can arise if the curve has disconnecting nodes. Tenni and the author in [10] considered singular curves, either reduced with planar singularities or lying on a smooth surface, and proved a generalisation of Clifford's theorem by studying rank 1 torsion-free

$\bowtie$ Marco Franciosi

marco.franciosi@unipi.it

1 Dipartimento di Matematica, Università di Pisa, Largo B. Pontecorvo 5, I-56127 Pisa, Italy 
sheaves of the form $\mathcal{I}_{S} \omega_{C}$, where $S$ is a zero-dimensional scheme and $\omega_{C}$ is the canonical sheaf.

In this paper, we consider reduced curves with planar singularities (e.g. semistable curves) and we study nef torsion-free sheaves of rank 1 whose degree is bounded from above by the degree of the canonical sheaf $\omega_{C}$. We remind that these curves are always Gorenstein.

Note that for a curve $C$ with many components the behaviour of the sections of a torsion-free sheaf and - as a consequence - of the Clifford index may be rather complicated. Nevertheless, it is possible to find an estimate for the Clifford index and to show some relations with the canonical ring of the curve. Indeed, given a reduced curve $C=C_{1} \cup \cdots \cup C_{n}$, and a rank 1 torsion-free sheaf $\mathcal{F}$ on $C$ such that

$$
0 \leq \operatorname{deg}\left(\mathcal{F}_{\mid C_{i}}\right) \leq \operatorname{deg}\left(\omega_{C \mid C_{i}}\right) \quad \forall C_{i}, i=1, \ldots, n
$$

we set $\operatorname{Cliff}(\mathcal{F}):=\operatorname{deg}(\mathcal{F})-2 h^{0}(C, \mathcal{F})+2$. Taking into account all the sheaves that contribute to the Clifford index (see Definition 3.5), we give the following definition of Clifford index for a reduced curve $C$

$$
\begin{aligned}
\operatorname{Cliff}(C):=\min \{\operatorname{Cliff}(\mathcal{F}): & \mathcal{F} \text { rank } 1 \text { torsion-free sheaf s.t. } \\
& \left.\mathcal{F} \text { verifies }(1) ; h^{0}(\mathcal{F}) \geq 2, h^{1}(\mathcal{F}) \geq 2\right\} .
\end{aligned}
$$

In Sect. 3, we prove that this minimum does exist and we show lower and upper bound for such index, investigating its relation with the combinatorial properties of the curves, in particular $m$-connectedness. We remind that a curve $C$ is $m$-connected if $A \cdot B:=\operatorname{deg}\left(\omega_{C \mid B}\right)-$ $\left(2 p_{\mathrm{a}}(B)-2\right) \geq m$ for any proper decomposition $C=A \cup B$ (cf. [6, Sect. 3]).

More precisely, we prove firstly that $\operatorname{Cliff}(C)$ can be negative if $C$ is not 4-connected, bounded from below by $-n+1$, where $n$ is the number of irreducible components of $C$ (see Proposition 3.2) and in Example 3.12 we show that such bound is sharp providing examples given by chains of curves. Moreover, in Example 3.13 we exhibit a curve $C$ not 4-connected with canonical sheaf very ample but whose Clifford index is negative, showing that for a curve with many components the geometric meaning of the Clifford index is more subtle.

On the contrary, in Theorem 3.6 we prove that $\operatorname{Cliff}(C) \geq 0$ if $C$ is 4 -connected and in Theorem 3.14 we prove that for every invertible sheaf $\mathcal{L}$ one always has $\operatorname{Cliff}(\mathcal{L}) \geq 0$, independently from the connectedness of the curve. Finally, in Theorem 3.9 we point out the following constraints occasioned by the numerical connectedness of $C$ : if $C$ is $m$-connected but $(m+1)$-disconnected (that is, there is a decomposition $C=A \cup B$ with $A \cdot B=m)$ and it does not contain rational components, then

$$
\operatorname{Cliff}(C) \leq \min \left\{m-2,\left\lfloor\frac{p_{\mathrm{a}}(C)-1}{2}\right\rfloor\right\} .
$$

We remark that our results can still be applied to irreducible curves with planar singularities. In particular, for an irreducible curve $C$, one always has $\operatorname{Cliff}(C) \geq 0$, with equality holding iff $C$ is hyperelliptic.

To show that the Clifford index we have introduced above has a geometrical meaning, in Sect. 4 we give a proof of Green's conjecture for a $m$-connected curve obtained glueing together two smooth curves. More precisely, we consider a stable curve $C=C_{1} \cup C_{2}$ given by the union of an irreducible smooth general curve $C_{1}$ of genus $g_{1}$ and an irreducible smooth curve $C_{2}$ of genus $g_{2}$, meeting transversally in $m$ distinct points. For such a curve $C$, if $4 \leq m \leq \frac{g_{1}+1}{2}$ and $1 \leq g_{2}$, we show that Cliff $(C)=m-2$ and Green's conjecture holds for $C$, i.e. $\mathcal{K}_{p, 1}\left(C, \omega_{C}\right)=0$ iff $p \geq p_{a}(C)-\operatorname{Cliff}(C)-1$, where $\mathcal{K}_{p, 1}\left(C, \omega_{C}\right)$ denotes the $p$ th Koszul group with value in $\omega_{C}$ (see Green's paper [11]). This result is only a modest 
novelty, since it is based on fundamental results of Voisin in [19,20] and Aprodu in [1], but we hope it should be helpful in studying curves with many components, e.g. stable curves.

A second application of our results can be found in the paper [3], where the authors, in order to characterise Brill-Noether-Petri curves, analyse the Petri homomorphism for rank 2 vector bundles on a (not necessarily smooth) curve $C$ by using some results on the Clifford index.

\section{Notation and preliminary results}

We work over an algebraically closed field $\mathbb{K}$ of characteristic 0 .

Throughout this paper, $C=C_{1} \cup \cdots \cup C_{n}$ denotes a reduced curve with planar singularities. The $C_{i}$ 's are the irreducible components of $C$.

A subcurve $B \subseteq C$ is a curve of the form $B=C_{i_{1}} \cup \cdots \cup C_{i_{k}}$ with $\left\{i_{1}, \ldots, i_{k}\right\} \subseteq\{1, \ldots, n\}$. For every subcurve $B \subseteq C$, we denote by $\omega_{B}$ its canonical sheaf (see [14], Chap. III, Sect. $7)$, by $K_{B}$ a canonical divisor, that is, a Cartier divisor so that $\mathcal{O}_{B}\left(K_{B}\right) \cong \omega_{B}$, and by $p_{\mathrm{a}}(B)$ the arithmetic genus of $B, p_{\mathrm{a}}(B)=1-\chi\left(\mathcal{O}_{B}\right)$. Note that, by our assumptions, every $B \subseteq C$ is Gorenstein (i.e. $\omega_{B}$ is invertible.)

A decomposition $C=A \cup B$ means $A=C_{j_{1}} \cup \cdots \cup C_{j_{h}}, B=C_{i_{1}} \cup \cdots \cup C_{i_{k}}$ such that $\left\{j_{1}, \ldots, j_{h}\right\} \cup\left\{i_{1}, \ldots, i_{k}\right\}=\{1, \ldots, n\}$.

For a given decomposition $C=A \cup B$, we will use the following standard exact sequences:

$$
\begin{aligned}
& 0 \rightarrow \mathcal{O}_{A}(-B) \rightarrow \mathcal{O}_{C} \rightarrow \mathcal{O}_{B} \rightarrow 0, \\
& 0 \rightarrow \omega_{A} \rightarrow \omega_{C} \rightarrow \omega_{C \mid B} \rightarrow 0 .
\end{aligned}
$$

where $\mathcal{O}_{A}(-B) \cong \mathcal{I}_{A \cap B} \cdot \mathcal{O}_{A}$.

If $C=A \cup B$ is a decomposition of $C$, then the intersection product $A \cdot B$ is defined as follows:

$$
A \cdot B=\operatorname{deg}\left(\omega_{C \mid B}\right)-\left(2 p_{\mathrm{a}}(B)-2\right) .
$$

A curve $C$ is $m$-connected if $A \cdot B \geq m$ for every effective decomposition $C=A \cup B$ (cf. [6] for a detailed analysis on Gorenstein curves). An $m$-connected curve $C$ is said to be $(m+1)$-disconnected if there is a decomposition $C=A \cup B$ with $A \cdot B=m$.

For a decomposition $C=A \cup B$, we will use frequently the key formula

$$
p_{\mathrm{a}}(C)=p_{\mathrm{a}}(A)+p_{\mathrm{a}}(B)+A \cdot B-1 .
$$

Let $\mathcal{F}$ be a rank 1 torsion-free sheaf on $C$. For every subcurve $B \subseteq C$, the degree of $\mathcal{F}$ on $B$ can be defined by the formula $\operatorname{deg}\left(\mathcal{F}_{\mid B}\right)=\chi\left(\mathcal{F}_{\mid B}\right)-\chi\left(\mathcal{O}_{B}\right)$. A torsion-free sheaf $\mathcal{F}$ is said to be nef if $\operatorname{deg}\left(\mathcal{F}_{\mid B}\right) \geq 0$ for every $B \subseteq C$.

A cluster $S$ of degree $\operatorname{deg} S=r$ is a 0-dimensional subscheme with length $\mathcal{O}_{S}=$ $\operatorname{dim}_{\mathbb{K}} \mathcal{O}_{S}=r$. A cluster $S \subset C$ is subcanonical if the space $H^{0}\left(C, \mathcal{I}_{S} \omega_{C}\right)$ contains a generically invertible section, i.e. a section $s_{0}$ which does not vanish on any subcurve of $C$. Equivalently, $S$ is subcanonical if there exists an injective map $\mathcal{O}_{C} \hookrightarrow \mathcal{I}_{S} \omega_{C}$ (see [10, Sect 2.3]).

Given a subcanonical cluster $S$, we define its residual cluster $S^{*}$ with respect to a generic invertible section $s_{0} \in H^{0}\left(C, \omega_{C}\right)$ by the following exact sequence:

$$
0 \longrightarrow \mathcal{H o m}\left(\mathcal{I}_{S} \omega_{C}, \omega_{C}\right) \stackrel{\alpha}{\longrightarrow} \mathcal{H o m}\left(\mathcal{O}_{C}, \omega_{C}\right) \longrightarrow \mathcal{O}_{S^{*}} \longrightarrow 0
$$


where the map $\alpha$ is defined by $\alpha(\varphi): 1 \mapsto \varphi\left(s_{0}\right)$. See [10, Sect. 2] for the definition and main properties.

In the following theorem, we summarise some basic results proved in [6] on the relations of $m$-connectedness with the behaviour of the canonical sheaf $\omega_{C}$. For a general treatment, see [6, Sects. 2, 3] and [5].

Theorem 2.1 Let C be a Gorenstein curve, and let $\omega_{C}$ be the canonical sheaf of $C$. Then

(i) If $C$ is 1-connected, then $H^{1}\left(C, \omega_{C}\right) \cong \mathbb{K}$.

(ii) If $C$ is 2-connected and $C ¥ \mathbb{P}^{1}$, then $\left|\omega_{C}\right|$ is base point free. To be more precise, $P$ is a base point for $\left|\omega_{C}\right|$ if and only if there exists a decomposition $C=C_{1} \cup C_{2}$ such that $C_{1} \cdot C_{2}=1$ and $P$ is a smooth point for each $C_{i}$ satisfying $\omega_{C \mid C_{i}} \cong \omega_{C_{i}}(P)$.

(iii) If $C$ is 3-connected and $C$ is not honestly hyperelliptic (i.e. there does not exist a finite morphism $\psi: C \rightarrow \mathbb{P}^{1}$ of degree 2$)$, then $\omega_{C}$ is very ample.

(cf. [6, Theorems 1.1, 3.3, 3.6] and [5, Proposition 2.4]).

Remark 2.2 Note that for reduced curves the above implications are actually equivalences. Indeed, (i) is obvious; (ii) follows from the fact that a disconnecting point is necessarily a base point for $\left|\omega_{C}\right|$; (iii) follows since, given a decomposition $C=A \cup B$ with $A \cap B=\{P, Q\}$, then $\left|\omega_{C}\right|$ does not separate the 2 points. See also [5, Proposition 2.4] for a detailed analysis of the base points of $\left|\omega_{C}\right|$ on 2-disconnected curves.

\section{Clifford index of reduced curves}

\subsection{Clifford index of rank 1 torsion-free sheaves}

In this section, we extend the notion of Clifford index by taking into account nef rank 1 torsionfree sheaves whose multidegree is bounded from above by the degree of the canonical sheaf $\omega_{C}$.

Definition 3.1 Let $C=C_{1} \cup \cdots \cup C_{n}$ be a connected reduced curve with planar singularities and let $\mathcal{F}$ be a nef rank 1 torsion-free sheaf.

The Clifford index of $\mathcal{F}$ is

$$
\operatorname{Cliff}(\mathcal{F}):=\operatorname{deg}(\mathcal{F})-2 h^{0}(C, \mathcal{F})+2
$$

First of all, let us consider the case $\mathcal{F}=\mathcal{I}_{S} \omega_{C}$, where $S \subset C$ is a subcanonical cluster, i.e. $S$ is a zero-dimensional scheme such that $H^{0}\left(C, \mathcal{I}_{S} \omega_{C}\right)$ contains a generically invertible section.

Proposition 3.2 Let $C=C_{1} \cup \cdots \cup C_{n}$ be a connected reduced curve with planar singularities, and let $S$ be a subcanonical cluster. Then,

$$
\operatorname{Cliff}\left(\mathcal{I}_{S} \omega_{C}\right) \geq-n+1
$$

Proof We argue by induction on the number of irreducible components $n$.

If the curve $C$ is irreducible or reducible and 2-connected, it is a straightforward consequence of [10, Theorem 3.8].

If $C$ is connected but 2-disconnected, then we may take a decomposition $C=A \cup B$, with $\mathrm{A}, \mathrm{B}$ connected curves such that $A \cdot B=1$, i.e. $A \cap B=\{P\}$, a point which is smooth 
for both. Let $n_{A}$ be the number of irreducible components of $A$ and $n_{B}$ be the number of irreducible components of $B$, so that $n=n_{A}+n_{B}$.

Let $S$ be a subcanonical cluster, i.e. assume that $H^{0}\left(C, \mathcal{I}_{S} \omega_{C}\right)$ contains a section $s_{0}$ which does not vanish on any subcurve of $C$ and consider the intersection point $P$. Note that $P$ is a base point for the system $\left|\omega_{C}\right|$ by Theorem 2.1. Hence, without loss of generality, we may assume that $P \cap S \neq \emptyset$. Indeed, if this is not the case, we may consider a residual cluster $S^{*}$ with respect to $s_{0} \in H^{0}\left(C, \mathcal{I}_{S} \omega_{C}\right)$ (see $\S 2$ ). Since $P$ is a base point for $\left|\omega_{C}\right|$, then $P$ must intersect either $S$ or $S^{*}$. Serre duality implies that the Clifford index of $S$ and $S^{*}$ coincides (see [10, Remark 2.13]); thus, we may work with the cluster which contains $P$.

Since $P$ is a smooth point for both the curves and $C$ has planar singularities, we have the isomorphisms of invertible sheaves $\omega_{C \mid A} \cong \omega_{A}(P)$ and $\omega_{C \mid B} \cong \omega_{B}(P)$. Hence, being $P \cap$ $S \neq \emptyset$, there exists a cluster $T_{A}$ on $A$, resp. a cluster $T_{B}$ on $B$, such that $\left(\mathcal{I}_{S} \omega_{C}\right)_{\mid A} \cong \mathcal{I}_{T_{A}} \omega_{A}$, resp. $\left(\mathcal{I}_{S} \omega_{C}\right)_{\mid B} \cong \mathcal{I}_{T_{B}} \omega_{B}$. Note that they are subcanonical, since a generically invertible section in $H^{0}\left(C, \mathcal{I}_{S} \omega_{C}\right)$ restricts to a generically invertible section in $H^{0}\left(A, \mathcal{I}_{S} \omega_{C \mid A}\right)=$ $H^{0}\left(\mathcal{I}_{T_{A}} \omega_{A}\right)$, and similarly on $B$. Therefore, by induction, we may assume $\operatorname{Cliff}\left(\mathcal{I}_{T_{A}} \omega_{A}\right) \geq$ $-n_{A}+1$ and $\operatorname{Cliff}\left(\mathcal{I}_{T_{B}} \omega_{B}\right) \geq-n_{B}+1$.

We point out that $\operatorname{deg}\left(\mathcal{I}_{T_{A}} \omega_{A}\right)+\operatorname{deg}\left(\mathcal{I}_{T_{B}} \omega_{B}\right)=\operatorname{deg}\left(\mathcal{I}_{S} \omega_{C}\right)$. Consider now the MayerVietoris sequence

$$
0 \rightarrow \mathcal{I}_{S} \omega_{C} \rightarrow \underset{\mathcal{I}_{T_{B}} \omega_{B}}{\oplus} \stackrel{\mathcal{I}_{T_{A}} \omega_{A}}{\oplus} \rightarrow \mathcal{O}_{P} \rightarrow 0
$$

Firstly, assume that $H^{0}\left(A, \mathcal{I}_{T_{A}} \omega_{A}\right) \oplus H^{0}\left(B, \mathcal{I}_{T_{B}} \omega_{B}\right) \rightarrow \mathcal{O}_{P}$ is onto. Note that this holds when $\operatorname{Cliff}\left(\mathcal{I}_{T_{A}} \omega_{A}\right)$ is minimal since by [10, Lemma 2.19] the restriction map $H^{0}\left(A, \mathcal{I}_{T_{A}} \omega_{A}\right) \rightarrow \mathcal{O}_{P}$ is surjective. In this case, we have $h^{0}\left(C, \mathcal{I}_{S} \omega_{C}\right)=h^{0}\left(A, \mathcal{I}_{T_{A}} \omega_{A}\right)+$ $H^{0}\left(B, \mathcal{I}_{T_{B}} \omega_{B}\right)-1$; hence, a straightforward computation yields $\operatorname{Cliff}\left(\mathcal{I}_{S} \omega_{C}\right)$ $\geq \operatorname{Cliff}\left(\mathcal{I}_{T_{A}} \omega_{A}\right)+\operatorname{Cliff}\left(\mathcal{I}_{T_{B}} \omega_{B}\right)$. Therefore, by induction we have

$$
\operatorname{Cliff}\left(\mathcal{I}_{S} \omega_{C}\right) \geq \operatorname{Cliff}\left(\mathcal{I}_{T_{A}} \omega_{A}\right)+\operatorname{Cliff}\left(\mathcal{I}_{T_{B}} \omega_{B}\right) \geq-n_{A}-n_{B}+2=-n+2
$$

If the above map (5) is not surjective on global sections, then in particular $H^{0}\left(A, \mathcal{I}_{T_{A}} \omega_{A}\right) \rightarrow$ $\mathcal{O}_{P}$ is not onto; hence, we have that $\operatorname{Cliff}\left(\mathcal{I}_{T_{A}} \omega_{A}\right)$ is not minimal by [10, Lemma 2.19], i.e. by induction we may assume $\operatorname{Cliff}\left(\mathcal{I}_{T_{A}} \omega_{A}\right) \geq-n_{A}+2$.

Since in this case $h^{0}\left(C, \mathcal{I}_{S} \omega_{C}\right)=h^{0}\left(A, \bar{I}_{T_{A}} \omega_{A}\right)+h^{0}\left(B, \mathcal{I}_{T_{B}} \omega_{B}\right)$, then

$\operatorname{Cliff}\left(\mathcal{I}_{S} \omega_{C}\right) \geq \operatorname{Cliff}\left(\mathcal{I}_{T_{A}} \omega_{A}\right)+\operatorname{Cliff}\left(\mathcal{I}_{T_{B}} \omega_{B}\right)-2 \geq-n_{A}+2-n_{B}+1-2=-n+1$.

The following trivial remark will be useful in the proof of Theorem 3.4.

Remark 3.3 Let $C=A \cup B$ be an effective decomposition of $C$ in nontrivial subcurves. Let $S_{A} \subset A$ be a cluster such that $A \cap B \subset S_{A}$ and let $S_{B} \subset B$ be a cluster such that $A \cap B \subset S_{B}$. Consider an invertible sheaf $\mathcal{L}_{A}$ supported on $A$ and an invertible sheaf $\mathcal{L}_{B}$ supported on $B$. Then, the two rank 1 torsion-free sheaves $\mathcal{I}_{S_{A}} \mathcal{L}_{A}$ and $\mathcal{I}_{S_{B}} \mathcal{L}_{B}$ are supported, respectively, on $A$ and $B$ and the sheaf $\mathcal{I}_{S_{A}} \mathcal{L}_{A} \oplus \mathcal{I}_{S_{B}} \mathcal{L}_{B}$ is a rank 1 torsion-free sheaf on $C$, since the sheaves living on the two curves can be glued together as they both vanish on the intersection.

Theorem 3.4 Let $C=C_{1} \cup \cdots \cup C_{n}$ be a connected reduced curve with planar singularities. Then, the following numbers exist and coincide: 
(1) $\min \{\operatorname{Cliff}(\mathcal{F}): \mathcal{F}$ rank 1 torsion-free sheaf s.t.

$0 \leq \operatorname{deg}\left(\mathcal{F}_{\mid C_{i}}\right) \leq \operatorname{deg} \omega_{C \mid C_{i}}$ for $i=1, \ldots, n$;

$\left.h^{0}(\mathcal{F}) \geq 2, h^{1}(\mathcal{F}) \geq 2\right\}$

(2) $\min \left\{\operatorname{Cliff}\left(\mathcal{I}_{S} \omega_{C}\right): S \subset C\right.$ subcanonical cluster s.t.

$$
\left.h^{0}\left(C, \mathcal{I}_{S} \omega_{C}\right) \geq 2, h^{1}\left(C, \mathcal{I}_{S} \omega_{C}\right) \geq 2\right\}
$$

Proof By Proposition 3.2, the second minimum exists. It is moreover obvious that the second set is included in the first; thus, such minimum is bigger than or equal to the infimum of the first set.

To conclude the proof, it is enough to prove that for every rank 1 torsion-free sheaf $\mathcal{F}$ in the first set attaining the minimal Clifford index, there exists a subcanonical cluster $T$ such that $\mathcal{I}_{T} \omega_{C} \cong \mathcal{F}$. This is equivalent to proving that a rank 1 torsion-free sheaf $\mathcal{F}$ with minimal Clifford index is generically invertible and moreover there exists an inclusion $\mathcal{F} \hookrightarrow \omega_{C}$ which is generically surjective.

For the first statement, assume for a contradiction that $\mathcal{F}$ itself is not generically invertible and let $B \subset C$ be the maximal subcurve of $C$ such that every section $s \in H^{0}(C, \mathcal{F})$ vanishes identically on $B$. Consider the decomposition $C=A \cup B$. Then, by the standard exact sequence

$$
0 \rightarrow \mathcal{F}_{\mid A}(-B) \rightarrow \mathcal{F} \rightarrow \mathcal{F}_{\mid B} \rightarrow 0
$$

we obtain

$$
\begin{gathered}
H^{0}\left(A, \mathcal{F}_{\mid A}(-B)\right) \cong H^{0}(C, \mathcal{F}) \\
0 \rightarrow H^{0}(B, \mathcal{F}) \rightarrow H^{1}\left(A, \mathcal{F}_{\mid A}(-B)\right) \rightarrow H^{1}(C, \mathcal{F}) \rightarrow H^{1}(B, \mathcal{F}) \rightarrow 0 .
\end{gathered}
$$

We take the sheaf $\mathcal{G} \cong \mathcal{F}_{\mid A}(-B) \oplus \mathcal{O}_{B}(A)(-A)$ (we write $\mathcal{O}_{B}(A)(-A)$ to emphasise that we are considering it as the kernel sheaf of the restriction map $\left.\mathcal{O}_{B}(A) \rightarrow \mathcal{O}_{A \cap B}\right)$. By Remark 3.3, $\mathcal{G}$ is a rank 1 torsion-free sheaf and moreover it verifies the inequalities $0 \leq \operatorname{deg}\left(\mathcal{G}_{\mid C_{i}}\right) \leq$ $\operatorname{deg}\left(\mathcal{F}_{\mid C_{i}}\right)$ for every $C_{i}$ since $\mathcal{F}_{\mid A}(-B)$ does not vanish on any subcurve of $A$.

Then, it is immediately seen that $\mathcal{O}_{C} \hookrightarrow \mathcal{G}$, i.e. $\mathcal{G}$ is generically invertible, and, since obviously $\mathcal{O}_{B}(A)(-A) \cong \mathcal{O}_{B}$, we obtain

$$
h^{0}(\mathcal{G})=h^{0}\left(B, \mathcal{O}_{B}\right)+h^{0}\left(A, \mathcal{F}_{\mid A}(-B)\right) \geq h^{0}\left(A, \mathcal{F}_{\mid A}(-B)\right)+1 .
$$

Therefore, by Eq. (6), we obtain $h^{0}(\mathcal{G}) \geq h^{0}(C, \mathcal{F})+1 \geq 3$.

Furthermore, by Eq. (7), we have

$$
h^{1}\left(A, \mathcal{F}_{\mid A}(-B)\right)=h^{1}(C, \mathcal{F})+h^{0}(B, \mathcal{F})-h^{1}(B, \mathcal{F}),
$$

that is,

$$
h^{1}(\mathcal{G})=h^{1}\left(B, \mathcal{O}_{B}\right)+h^{1}\left(A, \mathcal{F}_{\mid A}(-B)\right) \geq h^{1}(C, \mathcal{F})+\operatorname{deg}\left(\mathcal{F}_{\mid B}\right)+h^{0}\left(\mathcal{O}_{B}\right) \geq 3 .
$$

Hence, $\mathcal{G}$ is a torsion-free sheaf in the first set such that $\operatorname{deg}(\mathcal{G}) \leq \operatorname{deg}(\mathcal{F}), h^{0}(\mathcal{G})>h^{0}(\mathcal{F})$ and $h^{1}(\mathcal{G}) \geq h^{1}(\mathcal{F})$; in particular, $\operatorname{Cliff}(\mathcal{G})<\operatorname{Cliff}(\mathcal{F})$, which is absurd.

Therefore, we have $\mathcal{O}_{C} \hookrightarrow \mathcal{F}$. Now we show that $\mathcal{F} \hookrightarrow \omega_{C}$ and that such morphism is generically surjective. The dual sheaf $\mathcal{H o m}\left(\mathcal{F}, \omega_{C}\right)$ satisfies the same assumptions $\mathcal{F}$ does and by Serre duality has the same Clifford index. Hence, thanks to the previous step, we have an injection $\mathcal{O}_{C} \hookrightarrow \mathcal{H} \operatorname{om}\left(\mathcal{F}, \omega_{C}\right)$. In particular, $H^{0}\left(C, \mathcal{O}_{C}\right) \hookrightarrow H^{0}\left(C, \mathcal{H o m}\left(\mathcal{F}, \omega_{C}\right)\right)=$ $\operatorname{Hom}\left(\mathcal{F}, \omega_{C}\right)$, that is, there is a map from $\mathcal{F}$ to $\omega_{C}$ not vanishing on any component. By automatic adjunction ([6, Proposition 2.4]), we conclude that $\mathcal{F} \cong \mathcal{I}_{T} \omega_{C}$, for some suitable zero-dimensional scheme $T$. 


\subsection{Clifford index of curves}

The above theorem allows us to introduce the following notion of Clifford index for a reduced curve.

Definition 3.5 Let $C=C_{1} \cup \cdots \cup C_{n}$ be a connected reduced curve with planar singularities. The Clifford index of $C$ is

$$
\begin{aligned}
\operatorname{Cliff}(C):=\min \{\operatorname{Cliff}(\mathcal{F}): & \mathcal{F} \text { rank } 1 \text { torsion-free sheaf s.t. } \\
& 0 \leq \operatorname{deg}\left(\mathcal{F}_{\mid C_{i}}\right) \leq \operatorname{deg}\left(\omega_{C \mid C_{i}}\right) \text { for every } C_{i} ; \\
& \left.h^{0}(\mathcal{F}) \geq 2, h^{1}(\mathcal{F}) \geq 2\right\}
\end{aligned}
$$

As in the smooth case, we say that a rank 1 torsion-free sheaf $\mathcal{F}$ contributes to the Clifford index of the curve $C$ if $h^{0}(C, \mathcal{F}) \geq 2$ and $h^{1}(C, \mathcal{F}) \geq 2$.

For 4-connected curves, the Clifford index is always nonnegative as can be seen by the following result.

Theorem 3.6 If $C$ is a 4-connected reduced curve with planar singularities, then $\operatorname{Cliff}(C) \geq$ 0 , with equality holding if and only if $C$ is honestly hyperelliptic.

Proof By [10, Theorem B ], if $C$ is 4-connected, then for every rank 1 torsion-free sheaf $\mathcal{F}$ we have $h^{0}(C, \mathcal{F}) \leq \frac{\operatorname{deg} \mathcal{F}}{2}+1$.

Moreover, the above-mentioned theorem shows that if equality holds, then $\mathcal{F} \cong \mathcal{I}_{T} \omega_{C}$, where $T$ is a subcanonical cluster and, as in the smooth case, either $T \equiv 0, K_{C}$ or $C$ is honestly hyperelliptic and $T$ is a multiple of the honest $g_{2}^{1}$.

Corollary 3.7 If $C$ is an irreducible curve with planar singularities, then $\operatorname{Cliff}(C) \geq 0$ and it is 0 if and only if $C$ is hyperelliptic.

If $C$ has many components, numerical $m$-connectedness plays a relevant role in the computation of the Clifford index. Indeed, we have the following.

Proposition 3.8 Let $C=C_{1} \cup \cdots \cup C_{n}$ be an m-connected reduced curve with planar singularities.

Assume there exists a decomposition $C=A \cup B$ such that $A \cdot B=m, p_{a}(A) \geq 1$, $p_{a}(B) \geq 1$. Then, there exists an invertible sheaf $\mathcal{F}$ such that $\operatorname{Cliff}(\mathcal{F})=m-2, h^{0}(\mathcal{F}) \geq$ $2, h^{1}(\mathcal{F}) \geq 2$.

Proof Consider the decomposition $C=A \cup B$ with $A \cdot B=m, p_{a}(A) \geq 1, p_{a}(B) \geq 1$. Note that $A$ and $B$ are numerically connected by minimality of $m$ (see [10, Lemma 2.8]).

If $m=1$, then by Theorem 2.1 there exists a base point $P$ for the canonical system. Thus, $\operatorname{Cliff}\left(\mathcal{I}_{P} \omega_{C}\right)=-1$ and we may conclude.

From now on, we may assume that $m \geq 2$ and in particular that the system $\left|\omega_{C}\right|$ is base point free. Choose a generic $s \in H^{0}\left(A, \omega_{C \mid A}\right)$ and take the effective divisor $\Delta=\operatorname{div}(s)$. Since $\left|\omega_{C}\right|$ is base point free, $\Delta$ is the union of smooth points and moreover by our construction we may assume $\Delta \cap B=\emptyset$. Consider the invertible sheaf $\mathcal{F}:=\mathcal{O}_{C}(\Delta)$. We have

$$
\mathcal{F}_{\mid A} \cong \omega_{C \mid A}, \quad \mathcal{F}_{\mid B} \cong \mathcal{O}_{B}
$$


In particular, $\mathcal{F}(-B)_{\mid A} \cong \omega_{A}$ and we have the exact sequence

$$
\begin{aligned}
& 0 \rightarrow H^{0}\left(A, \omega_{A}\right) \rightarrow H^{0}(C, \mathcal{F}) \rightarrow H^{0}\left(B, \mathcal{O}_{B}\right) \rightarrow \\
& \rightarrow H^{1}\left(A, \omega_{A}\right) \rightarrow H^{1}(C, \mathcal{F}) \rightarrow H^{1}\left(B, \mathcal{O}_{B}\right) \rightarrow 0 .
\end{aligned}
$$

But $H^{0}(C, \mathcal{F})$ does not vanish on $B$ by our construction, hence

$$
\begin{aligned}
& h^{0}(C, \mathcal{F})=h^{0}\left(B, \mathcal{O}_{B}\right)+h^{0}\left(A, \omega_{A}\right)=1+p_{\mathrm{a}}(A) \geq 2 \\
& h^{1}(C, \mathcal{F})=h^{1}\left(B, \mathcal{O}_{B}\right)+h^{1}\left(A, \omega_{A}\right)=1+p_{\mathrm{a}}(B) \geq 2
\end{aligned}
$$

since both $A$ and $B$ are numerically connected.

Finally, by the above computation, we get

$$
\begin{aligned}
\operatorname{Cliff}(\mathcal{F}) & =\operatorname{deg}_{C}\left(\mathcal{O}_{C}(\Delta)\right)-2 h^{0}\left(C, \mathcal{O}_{C}(\Delta)\right)+2 \\
& =2 p_{\mathrm{a}}(A)-2+m-2 \cdot\left(1+p_{\mathrm{a}}(A)\right)+2=m-2 .
\end{aligned}
$$

As an immediate consequence, we obtain the following theorem.

Theorem 3.9 Let $C=C_{1} \cup \cdots \cup C_{n}$ be a connected reduced curve with planar singularities. Assume $C_{i} \neq \mathbb{P}^{1}$ for every $i=1, \ldots, n$.

If $C$ is $m$-connected but $(m+1)$-disconnected, then

$$
\operatorname{Cliff}(C) \leq \min \left\{m-2,\left\lfloor\frac{p_{\mathrm{a}}(C)-1}{2}\right\rfloor\right\} .
$$

Proof First of all, let us show that $\operatorname{Cliff}(C) \leq\left\lfloor\frac{p_{\mathrm{a}}(C)-1}{2}\right\rfloor$ by a degeneration argument.

With this aim, we point out that $\mathcal{E} x t^{1}\left(\omega_{C}, \mathcal{O}_{C}\right)$ is a torsion sheaf and $\mathcal{E} x t^{2}\left(\omega_{C}, \mathcal{O}_{C}\right)=0$ since $C$ is a reduced one-dimensional Gorenstein scheme with planar singularities. Moreover, we have $\omega_{C} \cong \Omega_{C}^{1}$ (cf. [18, Chap. IV]); hence, by the Grothendieck local-to-global spectral sequence for Ext, we obtain $\operatorname{Ext}^{2}\left(\Omega_{C}^{1}, \mathcal{O}_{C}\right)=0$. By standard arguments in deformation theory, this vanishing implies that there are no obstructions to deform $C$ (see, e.g. [17, Proposition 2.4.8, Ex. 2.4.9]).

Therefore, we can consider a one-parameter degeneration $f: X \rightarrow T$, where $X$ is a smooth surface and $T$ an affine curve, i.e. we can assume that $f$ is flat and proper and that there is a point $t_{0} \in T$ such that $f^{-1}\left(t_{0}\right):=C_{0} \cong C$, whilst for every $t \neq 0, f^{-1}(t):=C_{t}$ is a smooth curve of genus $g=p_{\mathrm{a}}(C)$. For each integer $d$, let Pic ${ }_{f}^{d}$ be the degree- $d$ relative Picard scheme of $f$ parameterizing invertible sheaves of degree $d$ on the fibres of $f$ (see, e.g. [13]). Then, for every invertible sheaf $\mathcal{F}$ in $\operatorname{Pic}_{f}^{d}$ we have $\operatorname{Cliff}\left(\mathcal{F}_{\mid C_{t}}\right) \leq\left\lfloor\frac{p_{\mathrm{a}}(C)-1}{2}\right\rfloor$. Since $h^{0}\left(\mathcal{F}_{\mid C_{t}}\right)$ and $h^{1}\left(\mathcal{F}_{\mid C_{t}}\right)$ are semicontinuous function in $t$, if $\mathcal{F}_{\mid C_{t}}$ contributes to the Clifford index for some $t$, then $\mathcal{F}_{\mid C_{0}}$ contributes to the Clifford index of $C_{0}$ and moreover by definition it is

$$
\operatorname{Cliff}\left(\mathcal{F}_{\mid C_{0}}\right) \leq \operatorname{Cliff}\left(\mathcal{F}_{\mid C_{t}}\right) \leq\left\lfloor\frac{p_{\mathrm{a}}(C)-1}{2}\right\rfloor .
$$

Now let us show that $\operatorname{Cliff}(C) \leq m-2$, where $m:=\min \{A \cdot B: C=A \cup B, A \neq$ $\emptyset, B \neq \emptyset\}$.

Take a proper decomposition $C=A \cup B$ with $A \cdot B=m$. Note that $p_{\mathrm{a}}(A) \geq 1, p_{\mathrm{a}}(B) \geq 1$ by our assumptions, and $A$ and $B$ are numerically connected by minimality of $m$ (see [10, Lemma 2.8]). Therefore, by Proposition 3.8, there exists an invertible sheaf $\mathcal{F}=\mathcal{O}_{C}(\Delta)$ which contributes to the Clifford index of $C$ and whose Clifford index verifies $\operatorname{Cliff}(\mathcal{F})=$ $m-2$. 
Remark 3.10 If we restrict our attention to stable curves, i.e. if we consider $\overline{\mathcal{M}}_{g}$, the moduli space of stable curves of genus $g=p_{\mathrm{a}}(C)$, it is worth mentioning that the Clifford index, as defined in Definition 3.5, is not the limit of the Clifford index of smooth curves. More precisely, if a curve $C$ is limit of smooth curves $C_{t}$ with $\operatorname{Cliff}\left(C_{t}\right) \leq \gamma$, then by semicontinuity we still have $\operatorname{Cliff}(C) \leq \gamma$, but the converse does not hold. Indeed, one can see that the locus of $m$-connected stable curves has codimension $m$ in $\overline{\mathcal{M}}_{g}$, and Theorem 3.9 shows that those curves have Clifford index at most $m-2$. On the contrary, one can consider $\mathcal{M}_{g, d}^{r}$, the locus consisting of smooth curves carrying a $g_{d}^{r}$. For suitable values of $r$ and $d$, such locus consists of smooth curves having Clifford index $\leq m-2$, and for small value of $m$, it has a far bigger codimension than $m$. Therefore, a simple dimension count shows that $\operatorname{Cliff}(C)$ may not coincide with the limit $\operatorname{Cliff}\left(C_{t}\right)$.

Remark 3.11 Let $C$ be a $m$-connected, but $(m+1)$-disconnected, reduced curve of arithmetic genus $p_{\mathrm{a}}(C)>0$, and let $C=A \cup B$ be a decomposition of $C$ in two connected curves of arithmetic genus $p_{\mathrm{a}}(A)$, respectively, $p_{\mathrm{a}}(B)$, such that $A \cdot B=m$.

If $m \leq p_{\mathrm{a}}(A)+p_{\mathrm{a}}(B)+2$, then it is $m-2 \leq\left\lfloor\frac{p_{\mathrm{a}}(C)-1}{2}\right\rfloor$. Therefore, it is easy to construct stable curves with given Clifford index just by taking $m$ satisfying the above relation.

\subsection{Examples of curves with negative Clifford index}

In this section, we are going to show two examples of curves having negative Clifford index. The first example shows that the inequality of Proposition 3.2 is sharp. The second example shows that for curves not 4-connected, the geometric interpretation of the Clifford index is more subtle.

Example 3.12 Let $C=\bigcup_{i=0}^{n} \Gamma_{i}$ be a chain of smooth curves $\Gamma_{i}$, i.e. $\Gamma_{i} \cdot \Gamma_{i+1}=1$, otherwise $\Gamma_{i} \cdot \Gamma_{j}$ vanishes.

$$
\Gamma_{0} \longrightarrow \Gamma_{1} \cdots \cdots \cdots \cdots \Gamma_{n-1}-\Gamma_{n}
$$

Assume that every $\Gamma_{i}$ has positive genus and set $S=\operatorname{Sing} C$.

Then, $h^{0}\left(C, \mathcal{I}_{S} \omega_{C}\right)=\sum_{i=1}^{n} p_{\mathrm{a}}\left(C_{i}\right)=p_{\mathrm{a}}(C)$. Therefore, we obtain

$$
\operatorname{Cliff}\left(\mathcal{I}_{S} \omega_{C}\right)=2 p_{\mathrm{a}}(C)-2-\operatorname{deg} S-2 h^{0}\left(C, \mathcal{I}_{S} \omega_{C}\right)+2=-n+1 .
$$

Note that in the above example every point in $S$ is a base point of $\left|\omega_{C}\right|$. Now, let us point out that this is not always the case. Indeed, if $C$ is 3-connected but not 4-connected (i.e. there exists a decomposition $C=A \cup B$ such that $A \cdot B=3$ ) then it might happen that $\operatorname{Cliff}(C)<0$ even thought $\omega_{C}$ is very ample and normally generated, as shown in the following example.

Example 3.13 Take $C=\bigcup_{i=0}^{5} \Gamma_{i}$ and suppose that $p_{\mathrm{a}}\left(\Gamma_{i}\right) \geq 2$ for every $i$. Suppose moreover that the intersection products are defined by the following dual graph, where the existence of the simple line means that the intersection product between the two curves is 1 .

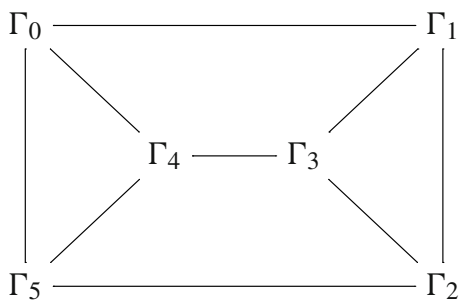


In this case, $\omega_{C}$ is very ample and normally generated by [9, Theorem 3.3].

For simplicity, assume moreover that $\Gamma_{i} \cap \Gamma_{j} \cap \Gamma_{k}=\emptyset$ for every $i, j, k$. Take $S=$ $\bigcup_{i, j}\left(\Gamma_{i} \cap \Gamma_{j}\right)$, which is a degree 9 cluster. Then, it is easy to check that $h^{0}\left(C, \mathcal{I}_{S} \omega_{C}\right)=$ $\oplus_{i=0}^{5} h^{0}\left(\Gamma_{i}, K_{\Gamma_{i}}\right)$, which yields $\operatorname{Cliff}\left(\mathcal{I}_{S} \omega_{C}\right)=-1$. (see [10, Example 5.2]).

\subsection{Clifford index of invertible sheaves}

The following theorem shows that the Clifford index of an invertible sheaf is always nonnegative.

Theorem 3.14 Let $C=C_{1} \cup \cdots \cup C_{n}$ be a reduced curve with planar singularities. Let $\mathcal{L}$ be an invertible sheaf such that $0 \leq \operatorname{deg}\left(\mathcal{L}_{\mid C_{i}}\right) \leq \operatorname{deg}\left(\omega_{C \mid C_{i}}\right)$ for $i=1, \ldots, n$. Then,

$$
h^{0}(C, \mathcal{L}) \leq \frac{1}{2} \operatorname{deg} \mathcal{L}+1, \text { i.e. } \operatorname{Cliff}(\mathcal{L}) \geq 0 .
$$

Proof First of all, we remark that we may assume $C$ to be connected since $h^{0}$ and deg are additive with respect to each connected component and that we may assume $\mathcal{L} \neq \mathcal{O}_{C}$, $\mathcal{L} \approx \omega_{C}$ and $h^{0}(C, \mathcal{L}) \neq 0$, since otherwise Eq. (8) is obvious.

Consider an invertible sheaf $\mathcal{L} \neq \mathcal{O}_{C}, \omega_{C}$ such that $0 \leq \operatorname{deg}\left(\mathcal{L}_{\mid C_{i}}\right) \leq \operatorname{deg}\left(\omega_{C \mid C_{i}}\right)$ for $i=1, \ldots, n$, and assume that $\operatorname{Cliff}(\mathcal{L})$ is minimal. Arguing as in the proof of Theorem 3.4 we conclude that $\mathcal{O}_{C} \hookrightarrow \mathcal{L} \hookrightarrow \omega_{C}$, i.e. there exists a subcanonical Cartier divisor $S$ such that $\mathcal{L} \cong \mathcal{I}_{S} \omega_{C}$ (see also [10, Sect. 2.3]). Hence, it is sufficient to show that for every subcanonical Cartier divisor $S$, one has $\operatorname{Cliff}\left(\mathcal{I}_{S} \omega_{C}\right) \geq 0$. We prove this result by induction on the number of irreducible components of $C$. To simplify the notation, we write $K_{C}-S$ for the divisor such that $\mathcal{O}_{C}\left(K_{C}-S\right) \cong \mathcal{I}_{S} \omega_{C}$.

If $C$ is irreducible, the classical Clifford's theorem holds (see [2, Sect. III:1], or see [10, Theorem A] for the singular case). If $C$ is 2-connected, the result follows from [10, Theorem A, case (a)].

Therefore, we are left to prove that Eq. (8) holds for reducible, connected but 2disconnected curves, i.e. we may assume that there exist connected subcurves $C_{1}$ and $C_{2}$ such that $C=C_{1} \cup C_{2}$ and $C_{1} \cap C_{2}$ consists of one single point $P$. In this case, $P$ is a smooth point for both curves and for $i=1,2$ we can write $K_{C \mid C_{i}} \equiv K_{C_{i}}+P$ as divisors on $C_{i}$.

Take the subcanonical Cartier divisor $S$. Arguing as in Proposition 3.2, we may assume that $P \cap S \neq \emptyset$ since otherwise we can take a residual Cartier divisor $S^{*}$.

Set $S_{1}:=S \cap C_{1}$ and $S_{2}:=S \cap C_{2}$. By the above argument, $P \cap S_{i} \neq \varnothing$ for $i=1,2$, and, since $P$ is a smooth point for each $C_{i}$, both the divisors $\left(S_{1}-P\right)$ and $\left(S_{2}-P\right)$ are Cartier and effective. Moreover, they are subcanonical on both the subcurves, since a generically invertible section in $H^{0}\left(C, K_{C}-S\right)$ restricts to a generically invertible section in $H^{0}\left(C_{i}, K_{C_{i}}\left(-\left(S_{i}-P\right)\right)\right)$.

The exact sequence (3) for the splitting $C=C_{1} \cup C_{2}$ can be written as follows:

$$
0 \rightarrow \omega_{C_{1}}\left(-S_{1}\right) \rightarrow \omega_{C}(-S) \rightarrow \omega_{C_{2}}\left(-\left(S_{2}-P\right)\right) \rightarrow 0 .
$$

In particular, it gives rise to the inequality

$$
h^{0}\left(C, K_{C}-S\right) \leq h^{0}\left(C_{1}, K_{C_{1}}-S_{1}\right)+h^{0}\left(C_{2}, K_{C_{2}}-\left(S_{2}-P\right)\right) .
$$

On $C_{2}$, we may apply our induction argument obtaining $h^{0}\left(C_{2}, K_{C_{2}}-\left(S_{2}-P\right)\right) \leq$ $\frac{1}{2} \operatorname{deg}\left(K_{C_{2}}-\left(S_{2}-P\right)\right)+1$. 
Now, let us consider $H^{0}\left(C_{1}, K_{C_{1}}-S_{1}\right)$. Counting dimensions, we have either that $h^{0}\left(C_{1}, K_{C_{1}}-S_{1}\right)=h^{0}\left(C_{1}, K_{C_{1}}-S_{1}+P\right)-1$ or that $h^{0}\left(C_{1}, K_{C_{1}}-S_{1}\right)=h^{0}\left(C_{1}, K_{C_{1}}-\right.$ $\left.\left(S_{1}-P\right)\right)$.

In the first case, Eq. (9) becomes

$$
h^{0}\left(C, K_{C}-S\right) \leq h^{0}\left(C_{1}, K_{C_{1}}-\left(S_{1}-P\right)\right)-1+h^{0}\left(C_{2}, K_{C_{2}}-\left(S_{2}-P\right)\right) .
$$

But $S_{1}-P$ and $S_{2}-P$ are subcanonical divisors on each subcurve; hence, we may apply induction on $C_{1}$ and $C_{2}$ obtaining

$$
\begin{aligned}
h^{0}\left(C, K_{C}-S\right) & \leq \frac{1}{2} \operatorname{deg}\left(K_{C_{1}}-\left(S_{1}-P\right)\right)+1-1+\frac{1}{2} \operatorname{deg}\left(K_{C_{2}}-\left(S_{2}-P\right)\right)+1 \\
& =\frac{1}{2} \operatorname{deg}\left(K_{C}-S\right)+1 .
\end{aligned}
$$

In the second case, $H^{0}\left(C_{1}, K_{C_{1}}-S_{1}\right) \cong H^{0}\left(C_{1}, K_{C_{1}}-\left(S_{1}-P\right)\right)$ and in particular $S_{1}$ is subcanonical on $C_{1}$ too. Therefore, we may apply induction on $C_{1}$ and $C_{2}$ and by Eq. (9) we get

$$
\begin{aligned}
h^{0}\left(C, K_{C}-S\right) & \leq h^{0}\left(C_{1}, K_{C_{1}}-S_{1}\right)+h^{0}\left(C_{2}, K_{C_{2}}-\left(S_{2}-P\right)\right) \\
& \leq \frac{1}{2} \operatorname{deg}\left(K_{C_{1}}-S_{1}\right)+1+\frac{1}{2} \operatorname{deg}\left(K_{C_{2}}-\left(S_{2}-P\right)\right)+1 \\
& =\frac{1}{2} \operatorname{deg}\left(K_{C}-S\right)+\frac{3}{2} .
\end{aligned}
$$

To conclude the proof, it is enough to show that the above inequality is strict. We argue by contradiction. Assume that $h^{0}\left(C, K_{C}-S\right)=\frac{1}{2} \operatorname{deg}\left(K_{C}-S\right)+\frac{3}{2}$. Then, necessarily

$$
\begin{aligned}
& h^{0}\left(C_{1}, K_{C_{1}}-S_{1}\right)=\frac{1}{2} \operatorname{deg}\left(K_{C_{1}}-S_{1}\right)+1 ; \\
& h^{0}\left(C_{2}, K_{C_{2}}-\left(S_{2}-P\right)\right)=\frac{1}{2} \operatorname{deg}\left(K_{C_{2}}-\left(S_{2}-P\right)\right)+1 .
\end{aligned}
$$

In particular, deg $S_{1}$ must be even and deg $S_{2}$ must be odd. But we may switch the roles of $C_{1}$ and $C_{2}$ and conclude that $\operatorname{deg} S_{2}$ is even and $\operatorname{deg} S_{1}$ is odd, which is clearly a contradiction.

Remark 3.15 The above result can be extended to nonreduced curves, under suitable assumptions. Indeed, the above theorem holds for 2-connected curves, whilst in the 2-disconnected case the key point of the proof is the existence of a decomposition $C=C_{1} \cup C_{2}$ with $C_{1} \cdot C_{2}=1$ such that:

(a) $C_{1}$ and $C_{2}$ satisfy Clifford's inequality;

(b) $P=C_{1} \cap C_{2}$ is a base point for $\left|K_{C}\right|$ and $P$ is a smooth point on $C_{i}$.

In order to use point (a), we do not really need that $C_{i}$ are reduced, just that they satisfy Clifford's inequality for some reason. E.g. 2-connected (possibly nonreduced) curves are perfectly fine.

In order to deal with point (b), the key fact is that by Theorem $2.1 P$ is a base point for $\left|\omega_{C}\right|$ if and only if there exists a subcurve $B \subset C$ such that $\omega_{C \mid B} \cong \omega_{B}(P)$ and $P$ is smooth for $B$.

\section{Green's conjecture for certain classes of reduced $m$-connected curves}

Let $C$ be a reduced curve, let $\mathcal{H}$ be an invertible sheaf on $C$ and let $W \subseteq H^{0}(C, \mathcal{H})$ be a subspace which yields a base point free system of projective dimension $r$. 
The Koszul groups $\mathcal{K}_{p, q}(C, \mathcal{H}, W)$ are defined as the cohomology at the middle of the complex

$$
\bigwedge^{p+1} W \otimes H^{0}\left(\mathcal{H}^{q-1}\right) \longrightarrow \bigwedge^{p} W \otimes H^{0}\left(\mathcal{H}^{q}\right) \longrightarrow \bigwedge^{p-1} W \otimes H^{0}\left(\mathcal{H}^{q+1}\right)
$$

If $W=H^{0}\left(C, \mathcal{H}\right.$ ), they are usually denoted by $\mathcal{K}_{p, q}(C, \mathcal{H})$ (see [11] for the definition and main results). The groups $\mathcal{K}_{p, q}(C, \mathcal{H})$ play a significant role if $\mathcal{H}$ is very ample and normally generated since in this case $\mathcal{K}_{p, q}(C, \mathcal{H}) \otimes \mathcal{O}_{\mathbb{P}^{n}}(-p-q)$ are the terms of the resolution of the ideal sheaf of the embedded curve (see [11, Theorem 2.a.15]).

If $C$ is a Gorenstein curves with planar singularities, 3-connected and not (honestly) hyperelliptic, then by [9, Theorem 3.3] $\omega_{C}$ is very ample and normally generated. Therefore, it is worth studying the Koszul groups $\mathcal{K}_{p, q}\left(C, \omega_{C}\right)$. Indeed, we have the following result.

Theorem 4.1 Let $C$ be a Gorenstein curve of arithmetic genus $p_{\mathrm{a}}(C) \geq 3$, with planar singularities, 3-connected and not honestly hyperelliptic. Then,

- $\mathcal{K}_{0, q}\left(C, \omega_{C}\right)=0$ for all $q>0$, i.e. $\omega_{C}$ is normally generated;

- $\mathcal{K}_{p, q}\left(C, \omega_{C}\right)=0$ if $q \geq 4$;

- $\mathcal{K}_{p, 3}\left(C, \omega_{C}\right) \cong \mathbb{C}$ if $p=g-2$, and $\mathcal{K}_{p, 3}\left(C, \omega_{C}\right)=0$ if $p \neq g-2$;

- $\mathcal{K}_{p, 1}\left(C, \omega_{C}\right)^{\vee} \cong \mathcal{K}_{g-p-2,2}\left(C, \omega_{C}\right)$;

- $\mathcal{K}_{p, 1}\left(C, \omega_{C}\right)=0 \Rightarrow \mathcal{K}_{p^{\prime}, 1}\left(C, \omega_{C}\right)=0 \quad \forall p^{\prime} \geq p$;

- $\mathcal{K}_{p, 2}\left(C, \omega_{C}\right)=0 \Rightarrow \mathcal{K}_{p^{\prime}, 2}\left(C, \omega_{C}\right)=0 \quad \forall p^{\prime} \leq p$.

Proof $\mathcal{K}_{0, q}\left(C, \omega_{C}\right)=0$ for all $q>0$ follows by [9, Theorem 3.3]. The remaining statements follow from the same the arguments used for smooth curves (see [11, Theorem 4.3.1]) and by the duality results given in [8, Proposition 1.4].

Taking Definition 3.5 for a generalisation of the usual Clifford index, Green's Conjecture ( [11, Conjecture 5.1] can be formulated without changes, i.e. given a 4-connected not hyperelliptic Gorenstein curve $C$, then one may ask whether

$$
\mathcal{K}_{p, 1}\left(C, \omega_{C}\right)=0 \stackrel{?}{\Longleftrightarrow} p \geq p_{a}(C)-\operatorname{Cliff}(C)-1 .
$$

First of all, we show that, as in the smooth case, one of the implications holds.

Proposition 4.2 (Green-Lazarsfeld) Let $C=C_{1} \cup \cdots \cup C_{n}$ be a 4-connected, not honestly hyperelliptic, reduced curve with planar singularities. Assume $C_{i} \neq \mathbb{P}^{1}$ for every $i=1, \ldots, n$.

Then,

$$
p \leq p_{\mathrm{a}}(C)-\operatorname{Cliff}(C)-2 \Longrightarrow \mathcal{K}_{p, 1}\left(C, \omega_{C}\right) \neq 0
$$

Proof First of all, let us point out that $\omega_{C}$ is normally generated by Theorem 4.1 and Cliff $(C) \geq 0$ by Theorem 3.6.

Take a proper subcanonical cluster $S$ such that $\mathcal{I}_{S} \omega_{C}$ computes the Clifford index of $C$ (cf. Theorem 3.4). Consider a generic invertible section $s_{0} \in H^{0}\left(C, \omega_{C}\right)$, and let $\Lambda:=\operatorname{div}\left(s_{0}\right)$ be the effective divisor corresponding to $s_{0}$ and $S^{*}$ be the residual cluster of $S$ with respect to $s_{0}$. Now, by [10, Sect. 2], we have the following exact sequence:

$$
0 \rightarrow \mathcal{O}_{C} \cong \mathcal{I}_{\Lambda} \omega_{C} \rightarrow \mathcal{I}_{S} \omega_{C} \rightarrow \mathcal{O}_{S^{*}} \rightarrow 0 .
$$


Therefore, we can consider $\mathbb{P}\left(H^{0}\left(\mathcal{I}_{S} \omega_{C}\right)\right)$ as a $g_{d}^{r}$, where $d=\operatorname{deg} \mathcal{I}_{S} \omega_{C}$ and $h^{0}\left(\mathcal{I}_{S} \omega_{C}\right)=$ $r+1$ and $\mathbb{P}\left(H^{0}\left(\mathcal{I}_{S^{*}} \omega_{C}\right)\right)$ as the residual $g_{d^{\prime}}^{r^{\prime}}$, where $d^{\prime}=\operatorname{deg} \mathcal{I}_{S^{*}} \omega_{C}$ and $h^{0}\left(\mathcal{I}_{S^{*}} \omega_{C}\right)=r^{\prime}+1$. Setting

$$
W_{1}=\operatorname{Im}\left\{H^{0}\left(\mathcal{I}_{S} \omega_{C}\right) \hookrightarrow H^{0}\left(\omega_{C}\right)\right\}, \quad W_{2}=\operatorname{Im}\left\{H^{0}\left(\mathcal{I}_{S^{*}} \omega_{C}\right) \hookrightarrow H^{0}\left(\omega_{C}\right)\right\}
$$

and

$$
\bar{D}_{1}=\operatorname{Ann}\left(W_{1}\right) \subset H^{0}\left(C, \omega_{C}\right)^{\vee}, \quad \bar{D}_{2}=\operatorname{Ann}\left(W_{2}\right) \subset H^{0}\left(C, \omega_{C}\right)^{\vee}
$$

we can repeat verbatim the argument adopted by Green and Lazarsfeld in [11, Appendix] obtaining $\mathcal{K}_{r+r^{\prime}-1,1}\left(C, \omega_{C}\right) \neq 0$.

To conclude, it is enough to see that $r+r^{\prime}-1=p_{\mathrm{a}}(C)-\operatorname{Cliff}(C)-2$ since $d^{\prime}=$ $2 p_{\mathrm{a}}(C)-2-d$ and $\operatorname{Cliff}\left(\mathcal{I}_{S} \omega_{C}\right)=\operatorname{Cliff}\left(\mathcal{I}_{S^{*}} \omega_{C}\right)$ by Serre duality. The nonvanishing of $\mathcal{K}_{p, 1}\left(C, \omega_{C}\right)$ for every $p<p_{\mathrm{a}}(C)-\operatorname{Cliff}(C)-2$ follows from Theorem 4.1.

Corollary 4.3 Let $C=C_{1} \cup \cdots \cup C_{n}$ be a connected reduced curve with planar singularities. Assume $C_{i} \neq \mathbb{P}^{1}$ for every $i=1, \ldots, n$.

If $C$ is $m$-connected but $(m+1)$-disconnected, then $\operatorname{Cliff}(C) \leq m-2$ and $\mathcal{K}_{p, 1}\left(C, \omega_{C}\right) \neq$ 0 if $p \leq p_{\mathrm{a}}(C)-m$.

In the following theorem, we show that Green's conjecture holds in the particular case of a stable curve consisting of two smooth components intersecting in $m$ distinct points.

Theorem 4.4 Let $g_{1}, g_{2}, m$ be integers such that $4 \leq m \leq \frac{g_{1}+1}{2}$ and $g_{2} \geq 1$.

Let $C=C_{1} \cup C_{2}$ be a stable curve given by the union of an irreducible smooth general curve $C_{1}$ of genus $g_{1}$ and an irreducible smooth curve $C_{2}$ of genus $g_{2}$, meeting transversally in $m$ distinct points $\left\{x_{1}, \ldots, x_{m}\right\}$. Then,

$$
\operatorname{Cliff}(C)=m-2 \text { and } \mathcal{K}_{p, 1}\left(C, \omega_{C}\right)=0 \Longleftrightarrow p \geq p_{\mathrm{a}}(C)-\operatorname{Cliff}(C)-1 .
$$

Proof Since $p_{\mathrm{a}}(C)=g_{1}+g_{2}+m-1$, the theorem follows if we prove that $\operatorname{Cliff}(C)=m-2$ and $\mathcal{K}_{p, 1}\left(\omega_{C}\right)=0$ if and only if $p \geq g_{1}+g_{2}$.

First of all, note that by Theorem 2.1 the linear system $\left|\omega_{C}\right|$ yields an embedding $\varphi$ : $C \hookrightarrow \mathbb{P}^{p_{\mathrm{a}}(C)-1}$ such that $\varphi(C)$ is the union of two curves of genus $g_{1}$ (resp. $g_{2}$ ) and degree $2 g_{1}-2+m$ (resp. $2 g_{2}-2+m$ ) intersecting in $m$ points $\left\{\varphi\left(x_{1}\right), \ldots, \varphi\left(x_{m}\right)\right\}$.

For simplicity, we set $W:=H^{0}\left(C, \omega_{C}\right)$ and we denote by $S(W)$ the symmetric algebra on $W$.

Consider the standard exact sequence

$$
0 \rightarrow \mathcal{O}_{C_{2}}\left(-C_{1}\right) \rightarrow \mathcal{O}_{C} \rightarrow \mathcal{O}_{C_{1}} \rightarrow 0
$$

Twisting with $\omega_{C}^{\otimes q}$ and taking cohomology, we get the following exact sequence of $S(W)$ modules,

$$
\left.0 \rightarrow \bigoplus_{q \geq 0} H^{0}\left(C_{2},\left.\omega_{C}\right|_{\mid C_{2}} ^{\otimes q}\left(-C_{1}\right)\right) \rightarrow \bigoplus_{q \geq 0} H^{0}\left(C, \omega_{C}^{\otimes q}\right) \rightarrow \bigoplus_{q \geq 0} H^{0}\left(C_{1}, \omega_{C} \mid C_{1}\right)\right) \rightarrow 0 \text { (10) }
$$

where the maps preserve the grading.

We emphasise that $\varphi\left(C_{1}\right)$ and $\varphi\left(C_{2}\right)$ are embedded as degenerate curves in $\mathbb{P}\left(W^{\vee}\right)$, but we can still consider every term above as $S(W)$-modules. Therefore, we will use the notation $\mathcal{K}_{p, q}(-,-, W)$ to point out that we are finding the resolution of the ideal of such degenerate curves (see [11, Proof of Theorem (3.b.7)] for a similar argument). 
By [11, Corollary 1.4.d, Theorem 3.b.1], we have the following exact sequence of Koszul groups :

$$
\begin{aligned}
& \cdots \rightarrow \mathcal{K}_{p+1,0}\left(C_{1}, \omega_{C \mid C_{1}}, W\right) \rightarrow \mathcal{K}_{p, 1}\left(C_{2}, \mathcal{O}_{C_{2}}\left(-C_{1}\right), \omega_{C \mid C_{2}}, W\right) \rightarrow \\
& \rightarrow \mathcal{K}_{p, 1}\left(C, \omega_{C}\right) \rightarrow \mathcal{K}_{p, 1}\left(C_{1}, \omega_{C \mid C_{1}}, W\right) \rightarrow \cdots
\end{aligned}
$$

To deal with the above groups, we consider the splittings

$$
\begin{aligned}
& W=H^{0}\left(C, \omega_{C}\right)=H^{0}\left(C_{2}, \omega_{C \mid C_{2}}\right) \bigoplus U \text { with } U \cong H^{0}\left(C_{1}, \omega_{C_{1}}\right), \\
& W=H^{0}\left(C, \omega_{C}\right)=H^{0}\left(C_{1}, \omega_{C \mid C_{1}}\right) \bigoplus Z \text { with } Z \cong H^{0}\left(C_{2}, \omega_{C_{2}}\right) .
\end{aligned}
$$

Setting $s=\max \left\{0, p-g_{1}\right\}, t=\max \left\{0, p-g_{2}\right\}$, we have the following decompositions of the Koszul groups appearing in the above exact sequence:

$$
\begin{aligned}
\mathcal{K}_{p, 1}\left(C_{2}, \mathcal{O}_{C_{2}}\left(-C_{1}\right), \omega_{C \mid C_{2}}, W\right) & =\bigoplus_{s \leq p^{\prime} \leq p}\left[\mathcal{K}_{p^{\prime}, 1}\left(C_{2}, \mathcal{O}_{C_{2}}\left(-C_{1}\right), \omega_{C \mid C_{2}}\right) \otimes \bigwedge^{p-p^{\prime}} U\right] \\
\mathcal{K}_{p, 1}\left(C_{1}, \omega_{C \mid C_{1}}, W\right) & =\bigoplus_{t \leq p^{\prime \prime} \leq p}\left[\mathcal{K}_{p^{\prime \prime}, 1}\left(C_{1}, \omega_{C \mid C_{1}}\right) \otimes \bigwedge^{p-p^{\prime \prime}} Z\right]
\end{aligned}
$$

Let us study at first $\mathcal{K}_{p, 1}\left(C_{2}, \mathcal{O}_{C_{2}}\left(-C_{1}\right), \omega_{C \mid C_{2}}, W\right)$.

Fix $p^{\prime} \leq p$. By duality (cf. [8, Proposition 1.4]) and the shift properties of $\mathcal{K}_{p, q}$ (cf. [11, (2.a.17)]), we have the following isomorphisms

$$
\begin{aligned}
\mathcal{K}_{p^{\prime}, 1}\left(C_{2}, \mathcal{O}_{C_{2}}\left(-C_{1}\right), \omega_{C \mid C_{2}}\right) & \cong \mathcal{K}_{g_{2}+m-3-p^{\prime}, 1}\left(C_{2}, \omega_{C_{2}} \otimes \mathcal{O}_{C_{2}}\left(C_{1}\right), \omega_{C \mid C_{2}}\right) \\
& \cong \mathcal{K}_{g_{2}+m-3-p^{\prime}, 2}\left(C_{2}, \omega_{C \mid C_{2}}\right) .
\end{aligned}
$$

But $\operatorname{deg}\left(\omega_{C \mid C_{2}}\right)=2 g_{2}-2+m$. Hence, by [11, Theorem (4.a.1)]

$$
\mathcal{K}_{g_{2}+m-3-p^{\prime}, 2}\left(C_{2}, \omega_{C \mid C_{2}}\right)=0 \quad \text { if } \quad g_{2}+m-3-p^{\prime} \leq m-3 .
$$

Therefore, we get

$$
\mathcal{K}_{p, 1}\left(C_{2}, \mathcal{O}_{C_{2}}\left(-C_{1}\right), \omega_{C \mid C_{2}}, W\right)=0 \quad \text { if } \quad p \geq g_{1}+g_{2} .
$$

Now let us study $\mathcal{K}_{p, 1}\left(C_{1}, \omega_{C \mid C_{1}}, W\right)$.

By our assumption, $C_{1}$ is a general curve of genus $g_{1} \geq 2 m-1$. For a general curve of genus $g_{1}$, we have Cliff $\left(C_{1}\right)=\left\lfloor\frac{g_{1}-1}{2}\right\rfloor$ and by the results of Voisin on Green's conjecture for smooth curves with maximal Clifford index ( [19], [20]) we have $\mathcal{K}_{p, 1}\left(C_{1}, \omega_{C_{1}}\right)=0$ if $p \geq$ $g_{1}-\left\lfloor\frac{g_{1}+1}{2}\right\rfloor$.

By our construction $\omega_{C \mid C_{1}} \cong \omega_{C_{1}}\left(C_{2}\right) \cong \omega_{C_{1}} \otimes \mathcal{O}_{C_{1}}\left(x_{1}+\ldots+x_{m}\right)$, hence by the result of Aprodu on adjoint bundles [1, Theorem 3] we get

$$
\mathcal{K}_{p^{\prime \prime}, 1}\left(C_{1}, \omega_{C}\right)=0 \quad \text { if } \quad p^{\prime \prime} \geq g_{1}+m-\left\lfloor\frac{g_{1}+1}{2}\right\rfloor
$$

and in particular

$$
\mathcal{K}_{p, 1}\left(C_{1}, \omega_{C \mid C_{1}}, W\right)=0 \quad \text { if } \quad p \geq g_{2}+g_{1}+m-\left\lfloor\frac{g_{1}+1}{2}\right\rfloor .
$$


Therefore, since $m \leq \frac{g_{1}+1}{2}$ by our assumptions, we obtain

$$
\mathcal{K}_{p, 1}\left(C_{1}, \omega_{C \mid C_{1}}, W\right)=0 \quad \text { if } \quad p \geq g_{1}+g_{2} .
$$

Putting our vanishing results (12) and (13) into the exact sequence (11) we deduce that

$$
\mathcal{K}_{p, 1}\left(C, \omega_{C}\right)=0 \quad \text { if } \quad p \geq g_{1}+g_{2}=p_{\mathrm{a}}(C)-(m-2)-1 .
$$

To conclude the proof note that the above vanishing result implies $\operatorname{Cliff}(C) \geq m-2$ by Proposition 4.2, whereas we have $\operatorname{Cliff}(C) \leq m-2$ by Theorem 3.9 because $m-2 \leq$ $\left\lfloor\frac{p_{\mathrm{a}}(C)-1}{2}\right\rfloor$ by our numerical assumptions, that is, $\operatorname{Cliff}(C)=m-2$ and $\mathcal{K}_{p, 1}\left(C, \omega_{C}\right)=0$ if and only if $p \geq p_{\mathrm{a}}(C)-\operatorname{Cliff}(C)-1$.

Acknowledgements The author is grateful for support by the PRIN project 2015EYPTSB_010 "Geometry of Algebraic Varieties" of Italian MIUR. The author would like to thank Elisa Tenni for deep and stimulating discussions on these arguments and the anonymous referee for his/her interesting observations.

\section{References}

1. Aprodu, M.: On the vanishing of higher syzygies of curves. Math. Zeit. 241, 1-15 (2002)

2. Arbarello, E., Cornalba, M., Griffiths, P.A., Harris, J.: Geometry of Algebraic Curves. Vol. I, Fundamental Principles of Mathematical Sciences, vol. 267. Springer, Berlin (1985)

3. Arbarello, E., Bruno, A.: Rank-two vector bundles on Halphen surfaces and the Gauss-Wahl map for Du Val curves. J. Éc. Polytech. Math. 4, 257-285 (2017)

4. Caporaso, L.: Linear series on semistable curves. Int. Math. Res. Not. 2011(13), 2921-2969 (2011)

5. Catanese, F., Franciosi, M.: Divisors of small genus on algebraic surfaces and projective embeddings. In: Proceedings of the Hirzebruch 65 Conference on Algebraic Geometry (Ramat Gan, 1993), Israel Mathematical Conference Proceedings, vol. 9, American Mathematical Society, pp. 109-140 (1996)

6. Catanese, F., Franciosi, M., Hulek, K., Reid, M.: Embeddings of curves and surfaces. Nagoya Math. J. 154, 185-220 (1999)

7. Coppens, M., Martens, G.: Secant spaces and Clifford's theorem. Compos. Math. 78, 193-212 (1991)

8. Franciosi, M.: Adjoint divisors on algebraic curves. Adv. Math. 186, 317-333 (2004)

9. Franciosi, M., Tenni, E.: The canonical ring of a 3-connected curve. Rend. Lincei Mat. Appl. 25, 37-51 (2014)

10. Franciosi, M., Tenni, E.: On Clifford's theorem for singular curves. Proc. Lond. Math. Soc. 108(3), 225-252 (2014)

11. Green, M.: Koszul cohomology and the geometry of projective varieties. J. Differ. Geom. 19, 125-171 (1984). (Appendix with Robert Lazarsfeld)

12. Green, M., Lazarsfeld, R.: On the projective normality of complete linear series on an algebraic curve. Invent. Math. 83, 73-30 (1986)

13. Grothendieck, A.: Technique de descente et théorèmes d'existence en géométrie algébrique. V. Les schémas de Picard: théorèmes d'existence. Sém. Bourbaki 7(232), 143-161 (1995)

14. Hartshorne, R.: Algebraic Geometry. Graduate Texts in Mathematics, vol. 52. Springer, New York (1977)

15. Martens, H.H.: Varieties of special divisors on a curve. II. J. Reine Angew. Math 233, 89-100 (1968)

16. Martens, G.: Über den Clifford-Index algebraischer Kurven. J. Reine Angew. Math 336, 83-90 (1982)

17. Sernesi, E.: Deformations of Algebraic Schemes, Grundlehren der Mathematischen Wissenschaften 334. Springer, Berlin (2006)

18. Serre, J.-P.: Courbes Algébriques et Corps de Classes. Hermann, Paris (1959). (English translation, Springer, 1990)

19. Voisin, C.: Green's generic syzygy conjecture for curves of even genus lying on a $K 3$ surface. J. Eur. Math. Soc. 4(4), 363-404 (2002)

20. Voisin, C.: Green's canonical syzygy conjecture for generic curves of odd genus. Compos. Math. 141(5), $1163-1190(2005)$

Publisher's Note Springer Nature remains neutral with regard to jurisdictional claims in published maps and institutional affiliations. 